\title{
Productivity of authors in the field of diabetes: bibliographic analysis of trial publications
}

\author{
Frits Holleman, ${ }^{1}$ Mick Uijldert, ${ }^{1}$ Lennart F Donswijk, ${ }^{2}$ Edwin A M Gale ${ }^{3}$
}

'Department of Internal

Medicine F4-112, Academic

Medical Center, 1105AZ

Amsterdam, Netherlands

${ }^{2}$ Department of Internal

Medicine, Gelre Ziekenhuizen,

Apeldoorn, Netherlands

${ }^{3}$ Department of Diabetes and

Metabolism, University of

Bristol, Bristol, UK

Correspondence to:

F Holleman

f.holleman@amc.uva.nl

Additional material is published online only. To view please visit the journal online (http://dx.doi. org/10.1136/bmj.h2638)

Cite this as: BMJ 2015;350:h2638 doi: 10.1136/bmj.h2638

Accepted: 21 April 2015

\author{
ABSTRACT \\ OBJECTIVE \\ To determine whether trial publications of glucose \\ lowering drugs are dominated by a small group of \\ highly prolific authors ("supertrialists") and to identify \\ some of their characteristics.
}

DESIGN

Bibliographic analysis of trial publications.

DATA SOURCES AND REVIEW METHODS

We searched PubMed for all randomised controlled trials (RCTs) relating to glucose lowering drugs published between 1 January 1993 and 31 December 2013. From these publications we identified the 110 most prolific authors using PubReMiner. The 991 RCTs they published were examined for various characteristics such as author number, commercial sponsorship, company authorship, conflicts of interest, etc. The track record of the top 11 authors was studied in more detail.

\section{MAIN OUTCOME MEASURE}

Proportion of articles published by the top 110 and the top 11 authors.

\section{RESULTS}

3782 articles from 13592 authors were identified. The top 110 authors were named in 1227 (32.4\%) of all articles, and the top 11 authors in 397 (10.5\%) of all articles. The top 110 authors published 991 RCTs for a median of 20 (range 4-77) RCTs per author; the top 11 published 354 RCTs for a median of 42 (36-77) RCTs per author. Of the 110 top authors, 48 were employed by a pharmaceutical company. Of the 991 RCTs, 906 were commercially sponsored. Of 704 articles that could be

\section{WHAT IS ALREADY KNOWN ON THIS TOPIC}

Honorary authors (authors with little or no contribution to the work described) and ghost authors (professional writers whose contribution is not acknowledged) threaten the integrity of the evidence base in medicine

Honorary authorship is known to be more frequent in research articles than in reviews

Anecdotally, a few highly prolific authors with multiple conflicts of interest have appeared to dominate clinical trial publications, but this has not previously been quantified

\section{WHAT THIS STUDY ADDS}

This analysis shows that 110 highly prolific authors contributed to one third of the evidence base for glucose lowering treatment; of these, $44 \%$ were company employees and $56 \%$ were academics who work closely with the pharmaceutical companies

Eleven authors, including nine academics-here designated supertrialistscontributed $10 \%$ of the entire evidence base

This concentration of influence adds to concerns about the independence and integrity of the evidence base for treatment for diabetes. assessed for conflicts of interest, only 42 (6\%) were considered fully independent. Medical writing assistance was acknowledged in 439 (44.3\%) of 991 RCTs.

\section{CONCLUSION}

The past two decades have seen an explosive increase in the number of published clinical trials regarding glucose lowering treatment. Some authors have made a disproportionate contribution to the therapeutic evidence base; one third of the RCT evidence base on glucose lowering drug treatment for diabetes was generated by less than $1 \%$ of authors. Of these, $44 \%$ were company employees and $56 \%$ were academics who work closely with the pharmaceutical companies.

\section{Introduction}

The International Committee of Medical Journal Editors (ICMJE) has recommended that authorship of medical articles should be based on four criteria: (1) substantial contributions to the conception, design, data acquisition, data analysis, or data interpretation; (2) drafting or revising the article; (3) final approval of the version to be published; and (4) accountability for accuracy and integrity of the work discussed. ${ }^{1}$ The ICMJE recommends that all four criteria should be met, but authorship implies a substantial amount of work.

By specifying these criteria the ICMJE has aimed to improve the integrity of at least one aspect of the evidence base upon which clinical decision making rests. Despite this, a recent retrospective survey of honorary authorship (authors who do not meet authorship criteria) and ghost authorship (individuals who are not named as authors despite substantial contributions) found evidence of this in $21 \%$ of articles. ${ }^{2}$ Honorary authors were more frequently listed on research articles than on editorials or reviews.

The discovery of multiple new classes of agents for the treatment of diabetes ${ }^{3}$ has resulted in an explosion of research studies requiring frequent updates to treatment guidelines. These studies have for the most part been published in journals that subscribe to the ICMJE guidelines. Some authors, however, are far more active than others, to the extent that their names seem to pop out of every diabetes journal you open. To test this impression, we analysed the contribution of the most active authors in the diabetes community to the evidence base for glucose lowering drugs, and identified some factors associated with being a prolific author.

\section{Methods}

We searched PubMed for all randomised controlled trials (RCTs) published between 1 January 1993 and 31 December 2013 that focused on new drugs developed and subsequently marketed for the treatment of diabetes-insulin 
analogues, meglinitides, thiazolidinediones, glucagon-like peptide 1 (GLP-1) agonists, dipeptidyl peptidase 4 (DPP-IV) inhibitors, and sodium-glucose co-transporter-2 (SGLT-2) inhibitors. For these six groups all synonyms, subgroups, and individual generic drug names were collected and combined into one search strategy. We used an adapted form of the Cochrane Highly Sensitive Search Strategy to filter for RCTs. Animal studies were excluded. We limited ourselves to articles in the English language. The search strategy was constructed with the help of an experienced librarian. (See online appendix for the exact search.)

Our search yielded a total of 3782 RCTs by a total of 13592 authors (ultimate search date 1 April 2014). The distribution of the number of publications per author for the whole time period is shown in figure 1 .

Using PubReMiner (http://hgserver2.amc.nl/cgi-bin/ miner/miner2.cgi), a meta-tool for PubMed enabling the counting of the occurrence of certain search results (for example, author names) within a PubMed Search, lists of authors ranked by their number of publications were created for overlapping periods of three years with each period shifting by one year (1993-95; 1994-96; ......; 2011-13). The three year periods were chosen to identify persistently active authors rather than those who by some chance event had several articles published all in the same year but were not productive outside that single year. Separate rankings were made for total publications, publications as first author, and publications as last author. We chose to include these separate rankings for first and last authorship since these are presumably the positions of most influence. ${ }^{4}$

By creating separate lists by period we included not just the most productive authors overall but also the most productive authors within a certain period, thus correcting for the increase in publications over time.

\section{Top authors}

From 2001 onwards a clear increase in the number of publications per author was noted (fig 2). We therefore only took the period from 2001-13 into account when creating our lists of most productive authors. This resulted in 33 lists of authors, one for each of the 11 three year periods, in each of the three categories (any, first, or last authorship).

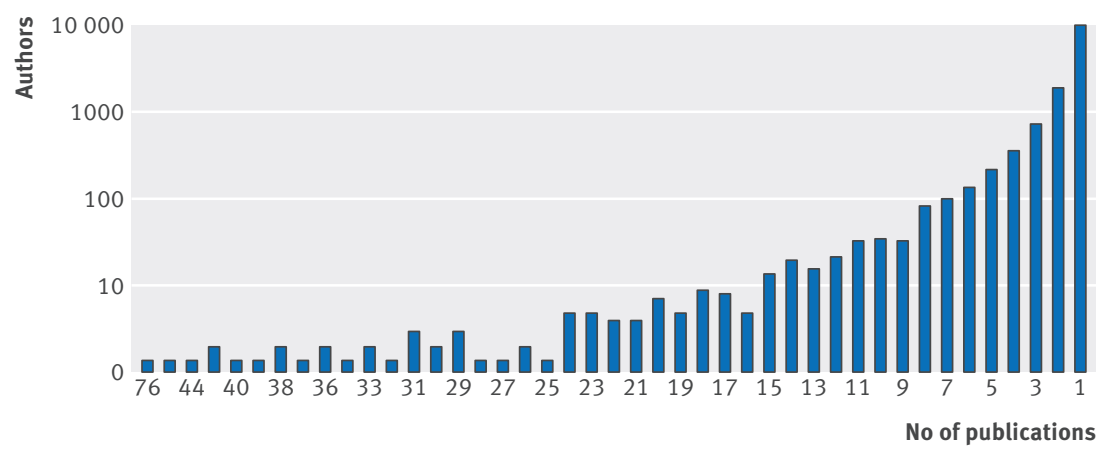

Fig 1 Distribution of authors by number of publications in the period 1993-2013. Based on the results of our primary search strategy (diabetes drug RCTs), in descending order of number of publications

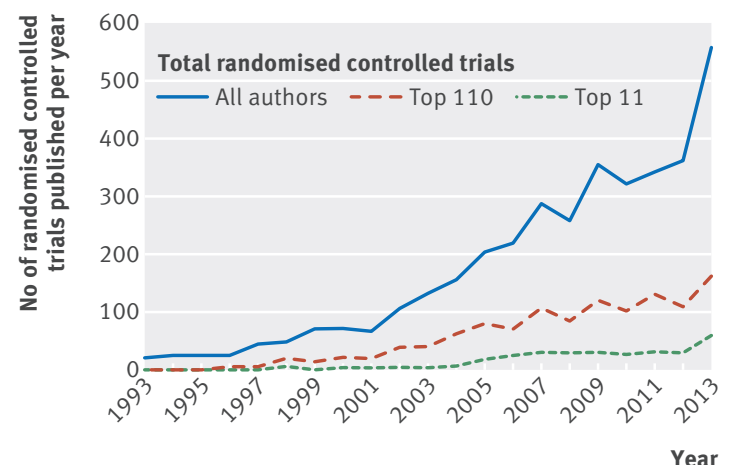

Fig 2 | Increase in publications over time. Based on our primary search strategy, the number of publications in each year is shown for all authors, the most productive 110 authors, and the most productive 11 authors

For the final total publications lists the top 20 authors in each three year period were selected and merged, and for the final first and last authors lists only the top five of each time period were merged. When authors tied for the 20th or 5th place, all were included in our list of most productive authors. Subsequently, authors were excluded from our list when article production was less than one RCT per year or less than four articles over a three year period. Manual correction was performed to eliminate duplicate authors, to differentiate between authors with the same surname and initials, and to create aggregate lists for the three categories.

The result was a list of 110 top authors. Of these, 84 authors qualified based on total publications. Of the remaining 26 authors, 10 qualified exclusively based on frequent first authorship and 16 on frequent last authorship.

\section{Article characteristics}

To identify the articles published by our top authors we combined the search strategy for the whole period from 1993 to 2013 with every individual author name of our top 110 authors. This yielded 1227 articles, 82 of which were not accessible through the University of Amsterdam Library on the 31 December 2013. A further 121 articles could not be classified as RCTs and 33 did not primarily concern glucose lowering drugs (fig 3). Thus, a total of 991 articles and their accompanying attachments or appendices were investigated further for the following: year of publication; journal name; sponsorship by a pharmaceutical company of the RCT, including name of this commercial sponsor; total number of authors; reported conflict of interests and contributions; percentage of pharmaceutical authors (authors employed by any pharmaceutical company); percentage of pharmaceutical authors and authors with conflict of interest in relation to the commercial sponsor; employment of the first, last, or corresponding author by the commercial sponsor; writing assistance (defined as editorial and writing assistance by individuals not listed as author); number of participants included; drugs studied (from the six main groups in our search string). 


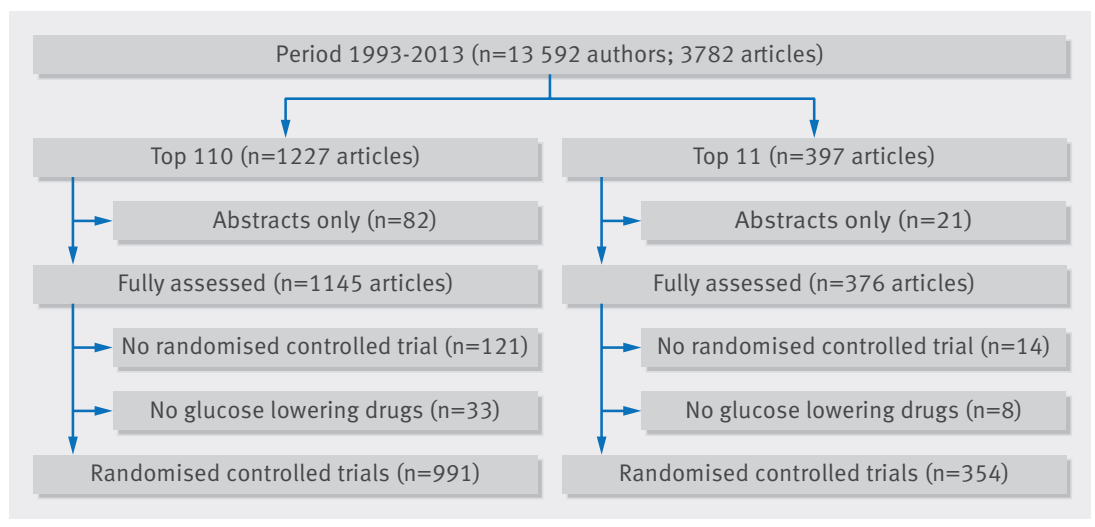

Fig 3 | Selection of randomised controlled trials published by most productive authors. While the top authors were selected based on their productivity between 2001 and 2013, all the articles they published between 1993 and 2013 were included. A separate selection was made for the subset of the 11 "supertrialists" in our top 110

\section{The supertrialists}

Of the top 110 authors, we aimed to select a top 10, but since two authors tied for the 10th position, we selected the 11 authors with the highest number of publications. A subset of 397 articles overall, and 354 (of 991) RCTs were attributable to the top 11 authors (fig 3).

For these top authors, an in-depth analysis was performed. Country of academic residence and total publication data available in PubMed including reviews, RCTs, RCTs of glucose lowering drugs, and regular publications were obtained, dating back to their first publication. Each author had a point in his or her career that can be described as a peak, the highest number of publications in a year. Reported conflicts of interest with pharmaceutical companies in that year and the three subsequent years were collected and summarised.

\section{Statistical analysis}

Simple descriptive statistics are given. Differences between groups were analysed with $\chi^{2}$ tests for categorical variables and Student's $t$ tests for continuous variables. SPSS Statistics for Windows, Version 21.0. (IBM Corp. Armonk, NY) software was used for analysis.

\section{Results}

General data 1993-2013

For the original study period of 1993-2013, a total of 13592 authors were involved in the publication of 3782 articles. Eighty six per cent of authors were involved in one or two articles; on the other hand, only 81 authors (less than $0.6 \%$ ) were involved in more than 15 articles during this whole period (fig 1). The average number of authors per article increased from 6.1 in 2001 to 7.8 in 2013.

There was a more than 20-fold increase in the yearly number of articles published over time, with a steep increase from 2001 onwards. However, the relative contribution of the top authors to the total literature remained fairly constant (fig 2).

\section{Top authors}

The top 110 authors were involved in 1227 (about one third) of all articles on glucose lowering drugs between
1993 and 2013. Disregarding those only qualifying on frequent first or last authorship, the top 84 authors by publication number were involved in 1039 articles. The top 11 authors were involved in 397 (about a 10th) of all articles.

On detailed analysis, the top 110 authors were involved in 991 RCTs for a median of 20 (range 4-77) RCTs per author; the top 84 were involved in 863 RCTs for a median of 24 (8-77); the top 11 were involved in 354 RCTs for a median of 42 (36-77) RCTs per author since 1993. A detailed breakdown of their individual activity in the past 10 years (2004-13) is shown in table 1.

Fifty three of our top 110 (and five of our top 11) authors came from the USA; $82 \%$ of our top 110 (and all of our top 11) came from just four countries: the USA, Italy, the UK, and Germany. Sixty two of our top 110 (and nine of our top 11) authors were affiliated with an academic institute; 48/110 and 2/11 were employed by a pharmaceutical company.

Of our top 110 authors, 41 authors published mainly ( $>75 \%$ of their articles) about one therapeutic agent. Of these, 38 were employed by a pharmaceutical company. Of the 42 authors who published studies of five or more different drugs, all were academic, non-pharma authors. This included all nine academic authors in our top 11.

From our top 110, the 62 authors from academic institutions occupied the first author position on 414 of 991 (42\%) articles and the last author position on 350 of 991 (35\%) articles. In total they occupied 1502 authorship positions on the 991 articles and, of these, 764 (51\%) were first or last authorships.

The nine academic authors in our top 11 claimed 395 authorship positions, with first authorship on 163/354 (46\%) articles and last authorship on 107/354 (30\%) articles.

The 48 authors employed by pharmaceutical companies were first author on 94 of 991 (9\%) articles and last author on 271 of 991 (27\%) articles. In total they occupied 913 authorship positions and of these 365 (40\%) were first or last authorships.

The two authors employed by pharmaceutical companies in our top 11 were first or last author on 3/354 (1\%) and 40/354 (11\%), respectively.

Two of nine academic authors in our top 11 (both from the same research group) never reported a conflict of interest; the other seven authors reported between eight and 21 conflicts of interest over time (median 16 conflicts of interest).

\section{Article characteristics}

The most popular journals for the 991 diabetes RCTs were Diabetes Care and Diabetes Obesity and Metabolism with 212 and 143 articles each. In all, 257 articles (26\%) reported studies with fewer than 50 participants, while 440 (44\%) articles reported on studies with more than 250 participants, suggesting that the majority of articles related to phase III and phase IV trials.

Of the 991 RCTs published by the top 110 authors, 906 (91\%) were commercially sponsored. 
Table 1 | Detailed breakdown of publications by the top 11 authors in the past 10 years. Supertrialists are identified by number and affiliation (academic or employee of pharmaceutical company) and ranked according to the number of diabetes randomised controlled trials (RCTs) written over the whole study period since 1993. Here only the data for the last 10 years are shown

No of publications by year

$\begin{array}{llllllllllll}\text { Author } & 2004 & 2005 & 2006 & 2007 & 2008 & 2009 & 2010 & 2011 & 2012 & 2013 & \text { Total }\end{array}$ No 1 Academic

\begin{tabular}{llllllllllll}
\hline All & 9 & 8 & 9 & 10 & 12 & 9 & 11 & 8 & 12 & $\mathbf{2 8}$ & 116 \\
\hline RCT & 6 & 4 & 8 & 5 & 10 & 7 & 11 & 6 & 10 & $\mathbf{2 0}$ & 87 \\
\hline DM & 4 & 3 & 7 & 4 & 5 & 6 & 8 & 6 & 8 & 16 & 67 \\
\hline
\end{tabular}

No 2 Pharma

\begin{tabular}{|c|c|c|c|c|c|c|c|c|c|c|c|}
\hline All & 1 & 5 & 1 & 8 & 16 & 5 & 6 & 7 & 4 & 6 & 59 \\
\hline RCT & 1 & 4 & 1 & 8 & 11 & 5 & 3 & 3 & 3 & 3 & 42 \\
\hline DM & 1 & 4 & 1 & 7 & 11 & 5 & 3 & 3 & 3 & 3 & 41 \\
\hline All & 3 & 0 & 4 & 2 & 3 & 2 & 4 & 12 & 15 & 34 & 79 \\
\hline $\mathrm{RCT}$ & 0 & 0 & 2 & 1 & 1 & 2 & 3 & 11 & 11 & 27 & 58 \\
\hline $\mathrm{DM}$ & 0 & 0 & 0 & 0 & 0 & 0 & 3 & 8 & 9 & 25 & 45 \\
\hline
\end{tabular}

No 4 Academic

\begin{tabular}{llllllllllll}
\hline All & 13 & 14 & 12 & 16 & 11 & 21 & 24 & 18 & 23 & $\mathbf{2 5}$ & 177 \\
\hline RCT & 10 & 10 & 8 & 11 & 9 & 11 & 16 & 12 & 16 & 19 & 122 \\
\hline DM & 1 & 5 & 5 & 4 & 2 & 5 & 4 & 4 & 6 & 5 \\
\hline
\end{tabular}

No 5 Academic

\begin{tabular}{llllllllllll}
\hline All & 12 & 11 & 15 & 20 & 13 & 14 & 14 & 14 & 15 & 15 & 143 \\
\hline RCT & 1 & 7 & 8 & 9 & 7 & 5 & 7 & 9 & 9 & 8 & 70 \\
\hline DM & 0 & 5 & 3 & 3 & 4 & 4 & 4 & 7 & 3 & 7 \\
\hline
\end{tabular}

No 6 Academic

\begin{tabular}{llllllllllll}
\hline All & 5 & 5 & 11 & 12 & 9 & 9 & 10 & 9 & 8 & 13 & 91 \\
\hline RCT & 2 & 3 & 7 & 6 & 3 & 6 & 5 & 2 & 3 & 6 & 43 \\
\hline DM & 2 & 3 & 5 & 6 & 3 & 5 & 3 & 2 & 1 & 3 & 33 \\
\hline No 7 Academic & & & & & & & & & & \\
\hline All & 16 & 11 & 19 & 19 & 24 & 20 & 20 & 22 & 11 & 28 & 190 \\
\hline RCT & 3 & 3 & 9 & 7 & 7 & 5 & 6 & 6 & 2 & 12 \\
\hline DM & 1 & 2 & 6 & 6 & 4 & 3 & 3 & 4 & 1 & 37
\end{tabular}

No 8 Academic

\begin{tabular}{llllllllllll}
\hline All & 11 & 12 & 15 & $\mathbf{2 1}$ & $\mathbf{2 1}$ & 17 & 13 & 15 & 14 & 15 & 154 \\
\hline RCT & 1 & 7 & 7 & 10 & $\mathbf{1 1}$ & 7 & 7 & 7 & 9 & 5 & 71 \\
\hline DM & 0 & 5 & 2 & 3 & 5 & 5 & 3 & 6 & 4 & 2 \\
\hline
\end{tabular}

No 9 Academic

\begin{tabular}{llllllllllll}
\hline All & 10 & 16 & 17 & 19 & 7 & 20 & 24 & 16 & 24 & $\mathbf{2 6}$ & 179 \\
\hline RCT & 7 & 8 & 9 & 11 & 5 & 11 & 12 & 8 & 10 & $\mathbf{1 5}$ & 96 \\
\hline DM & 1 & 5 & 5 & 4 & 2 & 5 & 3 & 3 & 4 & 5 \\
\hline
\end{tabular}

No 10 Academic

\begin{tabular}{llllllllllll}
\hline All & 15 & 20 & 14 & 22 & 16 & 27 & 32 & 18 & 16 & 18 & 198 \\
\hline RCT & 3 & 3 & 5 & 7 & 7 & 5 & 13 & 4 & 5 & 11 & 63 \\
\hline DM & 1 & 0 & 2 & 2 & 3 & 2 & 6 & 2 & 4 & 7 \\
\hline
\end{tabular}

No 11 Academic

\begin{tabular}{lllllllllllll}
\hline All & 12 & 15 & 10 & 16 & 8 & 11 & 4 & 8 & 3 & 7 & 94 \\
\hline RCT & 8 & 9 & 2 & 10 & 4 & 9 & 0 & 7 & 2 & 4 & 55 \\
\hline DM & 2 & 4 & 0 & 5 & 1 & 7 & 0 & 2 & 1 & 0 & 22 \\
\hline
\end{tabular}

Together

\begin{tabular}{llllllllllll}
\hline All & 107 & 117 & 127 & 165 & 140 & 155 & 162 & 147 & 145 & 215 & 1480 \\
\hline RCT & 42 & 58 & 66 & 85 & 75 & 73 & 83 & 75 & 80 & 130 & 767 \\
\hline DM & 13 & 36 & 36 & 44 & 40 & 47 & 40 & 47 & 44 & 80 & 427 \\
\hline
\end{tabular}

All=all publications; RCT=all RCTs; DM=all diabetes related RCTs published in a given year. The data in bold highlight the individual peaks in productivity.

Six of 991 RCTs did not clearly state author affiliations. Of the remaining 985 articles, 743 (75\%) had one or more authors employed by a pharmaceutical company and $684(69 \%)$ reported on the presence or absence of conflicts of interest. For an additional 20 articles, all authors were employed by a pharmaceutical company. Thus, a total of 704 articles could be assessed for conflict of interest. Only 6\% (42 out of 704) of articles could be considered fully independent (no commercial sponsor and no authors with reported conflicts of interest).

The articles that had pharmaceutical authors differed in many aspects from the articles where no pharmaceutical employees were involved. Results for the 985 articles by the top 110 authors are shown in table 2 . Disregarding those only qualifying on frequent first or 
Table 2 | Characteristics of articles with and without authors employed by a pharmaceutical company. Of 991 articles, six articles did not clearly state author affiliations and these were excluded from this analysis $(n=985)$

\begin{tabular}{|c|c|c|c|}
\hline & $\begin{array}{l}\text { No pharma authors } \\
(n=242)\end{array}$ & $\begin{array}{l}1 \text { pharma author } \\
(n=743)\end{array}$ & $\mathrm{P}$ \\
\hline Commercially sponsored trial & $159(65.7)$ & $742 / 743(99.9)$ & $<0.00^{*}$ \\
\hline Included patients/RCT & 666 & 743 & $0.597 \dagger$ \\
\hline Authors per article & 7.8 & 7.3 & $0.032 t$ \\
\hline Contributions reported & $46(19.0)$ & $202(27.2)$ & $0.011^{\star}$ \\
\hline Conflict of interest reported & $137(56.6)$ & $547(73.6)$ & $<0.001^{\star}$ \\
\hline $\begin{array}{l}\text { Mean \% of authors with conflict } \\
\text { of interest per article }\end{array}$ & $26 \%$ & $89 \%$ & $<0.001^{\star}$ \\
\hline Writing assistance & $25(10.3)$ & $413(55.6)$ & $<0.001^{\star}$ \\
\hline \multicolumn{4}{|c|}{$\begin{array}{l}\text { Data are no (\%) of articles or mean no of articles. } \\
{ }^{\star} \chi^{2} \text { test. } \\
+ \text { †tudent's } t \text { test. } \\
\text { ¥See text: this is based on evaluation of } 704 \text { articles: } 684 \text { articles which reported on conflicts of interest and } 20 \\
\text { articles where all authors were employed by a pharmaceutical company. }\end{array}$} \\
\hline
\end{tabular}

last authorship, the articles by the top 84 authors showed a similar pattern (data not shown).

In articles with at least 1 pharmaceutical author, an average of $89 \%$ of the authors on the article had a conflict of interest. This percentage was remarkably stable over time (data not shown).

The contributions of the various authors to the study were reported in only $25 \%$ of all RCTs.

Medical writing assistance was reported in 439/991 (44\%) of all RCTs; in 204 cases this was provided by the commercial sponsor and in 235 by a third party (writing bureau).

\section{Discussion}

The number of published articles relating to glucose lowering treatment per year rose from 22 in 1993 to 70 in 2001 and 566 in 2013. The burden of publication was however unevenly distributed, in that 110 authors contributed to a third of all published RCTs, whereas the remaining 13482 authors generally contributed one or two articles each. Over $80 \%$ of top authors came from just four countries (the USA, Italy, the UK, or Germany). Of the 110 authors, 48 were employed by the pharmaceutical companies, and we consider it appropriate for their contribution to be recognised. A select group of 11 authors (two company employees), here referred to as the supertrialists, were involved in $10 \%$ of all articles, equivalent to 42 publications each. At their most productive these supertrialists managed to publish between seven and 27 RCTs per year (of which between five and 25 related to glucose lowering treatment), thus becoming regular contributors to the diabetes journals and household names at diabetes meetings.

Our study has some limitations. Firstly, we restricted ourselves to studies relating to glucose lowering drugs and we cannot easily extrapolate these data to other areas of medicine, although we suspect that similar patterns would emerge. Secondly, we could not easily distinguish multiple articles arising from the same large trials, so some studies may be represented more than once in our database. Thirdly, we focused only on RCTs and thus may have underestimated the effort of our top authors. A quick glance showed that our supertrialists published between two and 13 times as many articles overall as RCTs, and several of our top 110 authors were involved in guideline committees as well-raising questions as to conflicts of interest. ${ }^{5}$

As anyone who has tried will testify, the design, execution, analysis, and reporting of RCTs (and these averaged around 700 patients each), is a tremendously labour intensive affair. Although only 25\% of the 991 articles reviewed complied with the ICMJE recommendation to list the individual contributions of each author, ${ }^{1}$ our top authors must have struggled to do so much work. Fortunately, at least one of the authors was employed by a pharmaceutical company in 75\% (743/985) of the articles and so could offer professional support. Given that over $90 \%$ of these trials were commercially sponsored one would expect that the majority of the work was done by company employees, so these may actually be under-represented in our authorship lists. Since pharmaceutical authors generally limited their output to a single drug, they could also provide specialised insight for supertrialists dealing with more than five different agents. First authorship implies a leading role in drafting a paper, and last authorship implies major responsibility for its contents, but 906 of 991 RCTs were commercially sponsored, and dedicated medical writing assistance was available for $44 \%$ of articles; some companies routinely acknowledged help from a writing company for each article. Since drafting the remaining $56 \%$ of articles would have represented a major demand upon the named authors, other forms of support may have been available. ${ }^{6}$

The effort invested by the top authors was not without reward. The 62 top authors from academic institutes had a better than $50 \%$ chance to feature as first or last authors on their RCTs (from an average of 7.4 authorship positions); for our nine academic supertrialists this chance rose to two in three, no doubt reflecting their stature in the diabetes community. Company employed authors had a reduced chance of first authorship (10\%), which might reflect a company preference to use well known clinicians as first authors. The median number of conflicts of interest for our nine academic supertrialists was 16, suggesting that they were rewarded in more ways than authorship alone.

It is easy to understand why pharmaceutical companies seek out the assistance of such well published academic authors, for these have remarkable experience of diabetes treatment and the planning, execution, and presentation of clinical trials. They understand the expectations of clinicians and, not coincidentally, they are gifted communicators. Their names, furthermore, carry the stamp of authority, for they are "famous for being well known." However, the integrity of the evidence base for glucose lowering drugs might suffer from the paucity of truly independent research and an over-reliance of the diabetes community on a few authors from a few countries who have substantial conflicts of interest.

In conclusion, despite an ever increasing number of RCTs, a select core of 110 authors has generated one third of the evidence base for our clinical decisions in 
diabetes over the past 20 years; 11 of these are responsible for $10 \%$ of the output. While commending their industry, we would suggest that journals publishing their work should provide more detail as to their individual contributions-in line with ICMJE recommendations-and that the burden of authorship should perhaps be distributed more equitably in future.

We gratefully acknowledge the help of Ingeborg Nagel, clinical librarian at the AMC-UvA in creating the search algorithm and for alerting us to the existence of PubReMiner.

Contributors: $\mathrm{FH}$ contributed to the conception, design, data analysis and interpretation, and drafting/revision of the manuscript. MU and LD contributed to the design, data acquisition, analysis and interpretation, and drafting/revision of the manuscript. EG contributed to data analysis and interpretation and to drafting/revision of the manuscript. FH had full access to all of the data in the study and takes responsibility for the integrity of the data and the accuracy of the data analysis. FH is the study guarantor.

Funding: No funding was received for this project.

Competing interests: All authors have completed the ICMJE uniform disclosure form at www.icmje.org/coi disclosure.pdf and declare: no support from any organisation for the submitted work; FH has received speaker fees and research support from Sanofi, MSD, Eli Lilly, AstraZeneca and Janssen-Cilag in the past three years; no other relationships or activities that could appear to have influenced the submitted work

Ethical approval: None required.

Data sharing: Datasets are available from the corresponding author on email request.
Transparency: FH affirms that the manuscript is an honest, accurate, and transparent account of the study being reported; that no important aspects of the study have been omitted; and that any discrepancies have been disclosed.

This is an Open Access article distributed in accordance with the Creative Commons Attribution Non Commercial (CC BY-NC 4.0) license, which permits others to distribute, remix, adapt, build upon this work non-commercially, and license their derivative works on different terms, provided the original work is properly cited and the use is noncommercial. See: http://creativecommons.org/licenses/by-nc/4.0/.

1 International Committee of Medical Journal Editors. The New ICMJE Recommendations (August 2013). www.icmje.org/news-andeditorials/new_rec_aug2013.html.

2 Wislar JS1, Flanagin A, Fontanarosa PB, Deangelis CD. Honorary and ghost authorship in high impact biomedical journals: a cross sectional survey. BMJ 2011;343:d6128

3 Nathan DM. Finding new treatments for diabetes - how many, how fast ... how good? N Engl/ Med 2007:356:437-40.

4 Wren JD, Kozak KZ, Johnson KR, Deakyne SJ, Schilling LM, Dellavalle RP. The write position. A survey of perceived contributions to papers based on byline position and number of authors. EMBO Rep 2007:8:988-91.

5 Lenzer I. Why we can't trust clinical guidelines. BMJ 2013:346:f3830.

6 Sismondo S. Ghost management: How much of the medical literature is shaped behind the scenes by the pharmaceutical industry? PLoS Med 2007:4:e286.

7 Ross JS, Hill KP, Egilman DS, Krumholz HM. Guest authorship and ghostwriting in publications related to rofecoxib. A case study of documents from rofecoxib litigation. JAMA 2008;299:1800-12.

๑) BMJ Publishing Group Ltd 2015

Supplementary material: appendix giving full search strategy 